\title{
A NEW CLASS OF GENERALIZED NONLINEAR MULTI-VALUED QUASI-VARIATIONAL-LIKE INCLUSIONS WITH $H$-MONOTONE MAPPINGS
}

\author{
YeOl JE CHO AND HeNG-YOU LAN
}

\begin{abstract}
In this paper, we introduce and study a new class of generalized nonlinear multi-valued quasi-variational-like inclusions with $H$-monotone operators in Hilbert spaces. By using the resolvent operator method associated with $H$-monotone operator due to Fang and Huang, we construct a new iterative algorithm for solving this kind of nonlinear multi-valued variational inclusions. We also prove the existence of solutions for the nonlinear multi-valued variational inclusions and the convergence of iterative sequences generated by the algorithm. Our results improve and generalize many known corresponding results.
\end{abstract}

Mathematics subject classification (2000): 49J40, 47J20, 65B05.

Key words and phrases: generalized nonlinear multi-valued variational inclusion with $H$-monotone operator, resolvent operator technique, relaxed cocoercive mapping, iterative algorithm with errors, existence and convergence.

\section{REFERENCES}

[1] S. ADLY, Perturbed algorithm and sensitivity analysis for a general class of variational inclusions, J. Math. Anal. Appl., 201, (1996), 609-630.

[2] R. AHMAD, Q. H. ANSARI, An iterative algorithm for generalized nonlinear variational inclusions, Appl. Math. Lett., 13, (5) (2000), 23-26.

[3] Y. J. CHO, J. H. KIM, N. J. HuANG AND S. M. KANG, Ishikawa and Mann iterative processes with errors for generalized strongly nonlinear implicit quasi-variational inequalities, Publ. Math. Debrecen, 58, (2001), 635-649.

[4] X. P. Ding, Perturbed proximal point algorithm for generalized quasivariational inclusions, J. Math. Anal. Appl., 210, (1) (1997), 88-101.

[5] X. P. DING, Generalized quasi-variational-like inclusions with nonconvex functionals, Appl. Math. \& Comput., 122, (3) (2001), 267-282.

[6] X. P. Ding, C. L. LuO, Perturbed proximal point algorithms for general quasi-variational-like inclusions, J. Comput. Appl. Math., 113, (2000), 153-165.

[7] Y. P. FANG, N. J. HUANG, H-monotone operator and resolvent operator technique for variational inclusions, Applied Mathematics and Computation, 145, (2003), 795-803.

[8] Y. P. FANG, N. J. HuANG, $H$-monotone operators and system of variational inclusions, Communications on Applied Nonlinear Analysis, 11, (2004), 93-101.

[9] A. HASSOUNI, A. MoudAFI, perturbed algorithm for variational inclusions, J. Math. Anal. Appl., 185, (3) (1994), 706-712.

[10] N. J. HUANG, Mann and Ishikawa type perturbed iterative algorithms for generalized nonlinear implicit quasi-variational inclusions, Computers Math. Appl., 35, (10) (1998), 1-7.

[11] N. J. HuANG, Generalized nonlinear variational inclusions with noncompact valued mappings, Appl. Math. Lett., 9, (3) (1996), 25-29.

[12] N. J. HuANG, Generalized nonlinear implicit quasivariational inclusion and an application to implicit variational inequalities, Z. Angew. Math. Mech., 79, (8) (1999), 569-575. 
[13] M. M. JIN, Perturbed algorithm and stability for strongly nonlinear quasi-variational inclusion involving H-monotone operators, Math. Inequal. Appl., 9, (4) (2006), 771-779.

[14] J. S. JUNG, C. H. MORALES, The Mann process for perturbed $m$-accretive opertators in Banach spaces, Nonlinear Anal. 46, (2) (2001), 231-243.

[15] H. Y. LAN, J. K. KIM AND N. J. HuANG, On the generalized nonlinear quasi-variational inclusions involving non-monotone set-valued mappings, Nonlinear Funct. Anal. \& Appl., 9, (3) (2004), 451-465.

[16] C. H. LEE, Q. H. ANSARI AND J. C. YAO, A perturbed algorithm for strongly nonlinear variational-like inclusions, Bull. Austral. Math. Soc., 62, (2000), 417-426.

[17] L. W. LIU, Y. Q. LI, On generalized set-valued variational inclusions, J. Math. Anal. Appl., 261, (1) (2001), 231-240.

[18] S. B. NALDER, Multi-valued contraction mappings, Pacific J. Math., 30, (1969), 475-488.

[19] Z. NANiewicZ, P. D. PANAgiotopoulos, Mathematical Theory of Hemi-variational Inequalities and Applications, Marcel Dekker, New York, 1995.

[20] E. ZEIDLER, Nonlinear Functional Analysis and its Applications II: Monotone Operators, SpringerVerlag, Berlin, 1985.

[21] M. A. NoOr, K. I. NoOR AND T. M. Rassias, Invitation to variational inequalities, in: Analysis, Geometry and Groups: A Riemann Legacy Volume, Hadronic, FL, (1993), 373-448.

[22] SALAHUDDIN, A. RAIS, Generalized multivalued nonlinear quasi-variational like inclusions, Nonlinear Anal. Forum, 6, (2) (2001), 409-416.

[23] S. H. ShIM, S. M. KANG, N. J. HuAng AND Y. J. ChO, Perturbed iterative algorithms with errors for completely generalized strongly nonlinear implicit quasivariational inclusions, J. Inequal. Appl., 5, (4) (2000), 381-395.

[24] R. U. VERMA, Nonlinear $H$-monotone variational inclusions and resolvent operator technique, Int. J. Pure \& Appl. Math. Sci., 2, (1) (2005), 53-57.

[25] R. U. VERMA, Generalized system for relaxed cocoercive variational inequalities and projection methods, Journal of Optimization Theory and Applications, 121, (2004), 203-210.

[26] R. U. VERMA, Partially relaxed cocoercive variational inequalities and auxiliary problem principle, Journal of Applied Mathematics and Stochatic Analysis, 17, (2) (2004), 143-148.

[27] George X. Z. YuAn, KKM Theory and Applications in Nonlinear Analysis, Marcel Dekker, New York, 1999. 